\title{
New stacked photodevices for signal multiplexing and demultiplexing applications in the visible spectrum
}

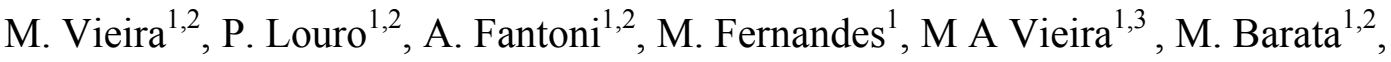 \\ ${ }^{1}$ Electronics Telecommunication and Computer Dept. ISEL, \\ R.Conselheiro Emídio Navarro, 1949-014 Lisboa, Portugal \\ e-mail:mv@isel.ipl.pt \\ ${ }^{2}$ CTS-UNINOVA' Quinta da Torre, 2829-516 Caparica, Portugal, \\ 3 CML-Traffic Department, Lisbon, Portugal
}

\begin{abstract}
Results on the use of a double a-SiC:H p-i-n heterostructure for signal multiplexing and demultiplexing applications in the visible range are presented. Pulsed monochromatic beams together (multiplexing mode), or a single polychromatic beam (demultiplexing mode) impinge on the device and are absorbed, accordingly to their wavelength. Red, green and blue pulsed input channels are transmitted together, each one with a specific transmission rate. The combined optical signal is analyzed by reading out, under different applied voltages, the generated photocurrent. Results show that in the multiplexing mode the output signal is balanced by the wavelength and transmission rate of each input channel, keeping the memory of the incoming optical carriers. In the demultiplexing mode the photocurrent is controlled by the applied voltage allowing regaining the transmitted information. A physical model supported by a numerical simulation gives insight into the device operation.
\end{abstract}

Keywords- Optical devices, a-SiC heterostructures, optical communication, multiplexing and demultiplexing applications over POF

\section{INTRODUCTION}

Recently polymer optical fibers (POF) are drawing the attention of industry since they are produced at very low cost and easily installed by non-specially trained people both in home networking, industrial control networks and cars applications [1]. Actual POF enabled products available in the market, use light emitting diodes (LED) for transmission light emission and photo diodes for its reception. Usually the available communication link is half duplex obliging to the use of two fibers when full duplex is needed. This approach is plausible when it corresponds to the less expensive one. Here wavelength division multiplexing (WDM), already in use in glass fiber technology, can contribute to the increase of the communication capacity of a POF infrastructure by allowing multiple simultaneous communication channels. In comparison to glass fibers POF offer easy and cost-efficient 978-1-4244-4346-8/09/\$25.00 C2009 IEEE processing and are more flexible for plug packing. POF can be passed with smaller radius of curvature and without any disruption because of larger diameter in comparison with glass fiber. These characteristics have opened possibilities for short distance communication systems. However, as the spectral window transmission lays within the visible spectrum the use of the WDM technique demands adequate optical devices for the multiplexing/demultiplexing operations. An example of preliminary work of WDM applied to POF fibers is presented in [2] where optical devices are used for light filtering.

WDM enables the use of a significant portion of the available fiber bandwidth by allowing many independent signals to be transmitted simultaneously, with each signal located at a different wavelength. Routing and detection of these signals can be accomplished independently, with the wavelength determining the communication path by acting as the signature of the origin, destination or routing. Components are therefore required to be wavelength selective. So, the conception of new devices for signal (de)multiplexing in the visible spectrum is a demand in this field $[3,4]$. Digital home appliance interfaces, home and car network and traffic control applications are foreseen due to the low cost associated to the a-SiC:H and POF technologies.

This paper presents results on the applicability of a multilayered a-SiC:H heterostructures as an electrically programmable optical filters for WDM transmission over POF. heterostructures as an electrically programmable optical filters for WDM transmission over POF. A physical model supported by a numerical simulation gives insight into the device operation.

\section{EXPERIMENTAL DETAILS.}

\section{A. Double-junction WDM device configuration}

The WDM device is a glass/ITO/a-SiC:H (p-i-n)/ a$\mathrm{SiC}: \mathrm{H}(-\mathrm{p}) / \mathrm{Si}: \mathrm{H}(-\mathrm{i}) / \mathrm{SiC}: \mathrm{H}$ (-n)/ITO double heterostructure 
produced by PECVD. The individual layers, the simplified test a-SiC:H p-i-n and a-Si:H p-i-n structures and the p-i-n/pi-n sensing element were fabricated by Plasma Enhanced Chemical Vapor Deposition at $13.56 \mathrm{MHz}$ radio frequency using a parallel-plate PECVD reactor. The substrate temperature was held at $300^{\circ} \mathrm{C}$, the power of the $\mathrm{RF}$ discharge at $20 \mathrm{~W}$, and the pressure in the deposition chamber was 270 mTorr for the p-type, 170 mTorr for the intrinsic and 180 mTorr for the n-type layers. Deposition conditions are described elsewhere [5]. The TCO contacts established for carrier collection are made of undoped $\mathrm{InOx}$ thin films deposited at room temperature by radio frequency plasma enhanced reactive thermal evaporation (rf-PERTE) of In [6].

In Figure 1 the device configuration is depicted. To enhance the light filtering properties of the device the thickness of the multilayer structure was optimized.
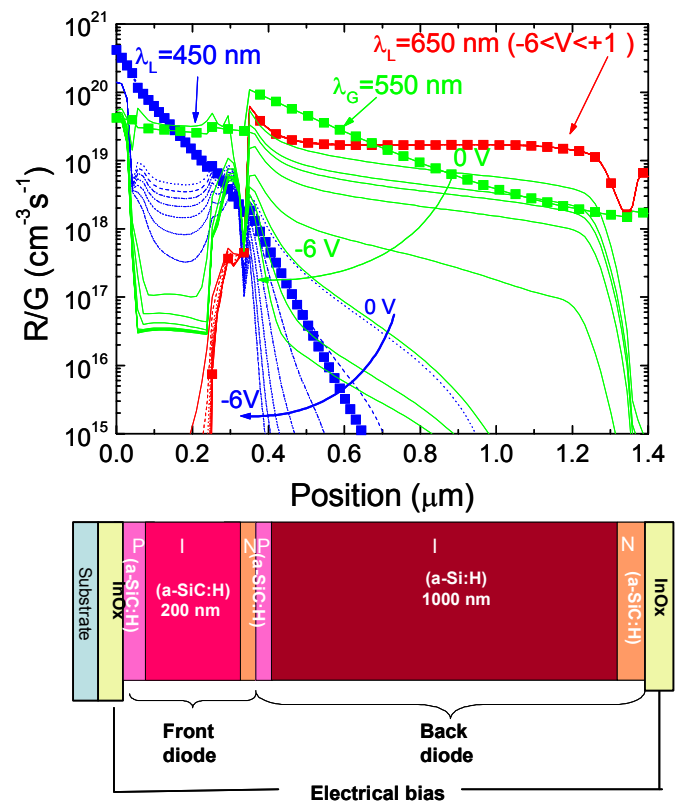

Figure 1 - a-SiC:H WDM device configuration. Recombination profiles (straight lines) under red $\left(\lambda_{R}=650 \mathrm{~nm}\right)$ green $\left(\lambda_{\mathrm{G}}=550 \mathrm{~nm}\right)$ and blue $\left(\lambda_{\mathrm{B}}\right.$ $=450 \mathrm{~nm})$ optical bias and different applied voltages $(-6 \mathrm{~V}<\mathrm{V}<0 \mathrm{~V})$. The generation profiles are also shown (symbols).

We used a device simulation program ASCA-2D [7] to simulate the generation and recombination profiles in the investigated structure under different light wavelength irradiations. Typical values of band tail and gap state parameters for amorphous materials were used. The doping level was adjusted in order to obtain approximately the same conductivity of the layers as in the tested samples. In the films the optical band gaps were chosen in compliance with the obtained experimental values. Band discontinuities were equally distributed over the valence and conduction band offsets $(\Delta \mathrm{Ev}=\Delta \mathrm{Ec}=0.15 \mathrm{eV})$. On the top of the figure it is displayed the recombination profiles (straight lines) under $\operatorname{red}\left(\lambda_{R}=650 \mathrm{~nm}\right)$ green $\left(\lambda_{\mathrm{G}}=550 \mathrm{~nm}\right)$ and blue $\left(\lambda_{\mathrm{B}}=450 \mathrm{~nm}\right)$ optical bias and different electrical bias $(-6 \mathrm{~V}<\mathrm{V}<0 \mathrm{~V})$. The generation profiles are also shown (symbols). Taking into account the simulated results, the thickness $(200 \mathrm{~nm})$ and the absorption coefficient of the deposited front photodiode are optimized for blue collection and red transmittance, and the thickness $(1000 \mathrm{~nm})$ of the back one adjusted to achieve full absorption in the greenish region and high collection in the red spectral one. As a result, both front and back diodes act as optical filters confining, respectively, the blue and the red optical carriers, while the green ones are absorbed across both [8].

\section{B. Optoelectronic characterizationof the single p- i-n photodiodes}

The p-i-n a-SiC:H used in these stacked devices was further analyzed in order to infer about the introduction of an $\mathrm{a}-\mathrm{SiC}: \mathrm{H}$ absorber on its optoelectronic properties. For this purpose a p-i-n a-SiC:H test structure $(200 \mathrm{~nm})$ and a p-i-n a$\mathrm{Si}: \mathrm{H}$ test diode $(1000 \mathrm{~nm})$ were deposited in the same conditions of the stacked device. Figure 2 shows the dark current-voltage characteristics of test $\mathrm{p}-\mathrm{i}(\mathrm{a}-\mathrm{SiC}: \mathrm{H})-\mathrm{n}$ and $\mathrm{p}$ $\mathrm{i}(\mathrm{a}-\mathrm{Si}: \mathrm{H})-\mathrm{n}$ photodiodes, measured at room temperature.

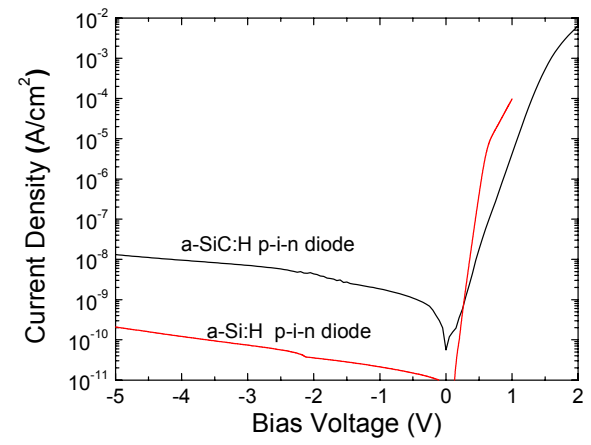

Figure $2-\mathrm{J}-\mathrm{V}$ characteristics measured in dark at room temperature of a-SiC:H p-i-n (200 nm) and a-Si:H p-i-n (500 nm) photodiodes.

As expected both diodes exhibit different current-voltage characteristics, under forward and reverse biases. From the linear region of the forward characteristic the diode ideality factor and the saturation reverse density current were extracted. It was observed respectively, for the a-SiC:H and a-Si:H p-i-n diodes, values of $80 \mathrm{pA} / \mathrm{cm} 2$ and $0.5 \mathrm{pA} / \mathrm{cm} 2$ for the saturation currents, and 3.65 and 1.45 for the ideality factors. For the a-Si:H based photodiode the observed parameters are similar to those reported in the literature [9]. In the a-SiC:H photodiode both parameters exhibit larger values, which are related to the higher defect density of the $\mathrm{a}-\mathrm{SiC}: \mathrm{H}$ material and probably to higher recombination rates. The exponential growth up to $1.4 \mathrm{eV}$ is due to the wider band gap of the intrinsic a-SiC:H. The optical band gap obtained from the Tauc's plot is $1.92 \mathrm{eV}$, which is $200 \mathrm{meV}$ higher than in a-Si:H. Under reverse biases the leakage current of the a-SiC:H diode is two orders of magnitude higher than in 
a-Si:H. This higher current density can be attributed to the carrier generation in the intrinsic layer and also to the defects in the i-p interface. We believe that the thermal generation in the intrinsic layer is the dominant leakage mechanism in device, because the voltage dependence of the reverse dark current is not strong (the dark current exhibits saturation). The a-SiC:H test structure was characterized by spectral response in the range $400 \mathrm{~nm}$ up to $700 \mathrm{~nm}$, under different optical and electrical biasing conditions. Figure 3 shows the spectral response of the test a-SiC: $\mathrm{H}$ p-i-n diode measured under reverse bias from $0 \mathrm{~V}$ to $-4 \mathrm{~V}$. No optical bias was superimposed.

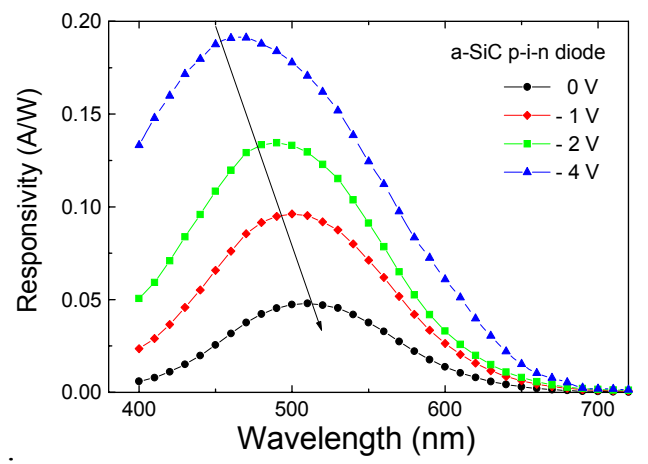

Figure 3 - Spectral response of the a-SiC:H p-i-n test diode.

If compared with the standard a-Si:H p-i-n diodes, results show that the spectral response dependence on the applied voltage is enhanced and that the maximum of the spectral sensitivity is shifted to lower wavelength values. This is due to the wider band gap of a-SiC:H that makes the device transparent to the red range of the visible spectrum. Besides, the photodiode sensitivity is dependent on the applied reverse bias. With the increasing bias voltage the spectral response maxima shift from $509 \mathrm{~nm}$ (at $0 \mathrm{~V}$ ) to $465 \mathrm{~nm}$ (at $4 \mathrm{~V}$ ), showing a voltage controlled dependence, which can be explained by lower mobility-lifetime product in comparison with a-Si:H.

\section{NUMERICAL SIMULATION}

Figure 4 reports the simulated electric field profile within the $\mathrm{p}-\mathrm{i}-\mathrm{n} / \mathrm{p}-\mathrm{i}-\mathrm{n}$ device for different wavelengths of the impinging light and for different values of the external electrical bias. Simulated results show that the shallow penetration of the blue photons into the front diode, the deep penetration of the red photons into the back absorber or the decay of the green absorption across both (Figure 1), controls the internal electrical field. The balance between the electrical field adjustments due to the non uniform absorption throughout the structures depends on the generation/recombination ratio profiles at each applied voltage. When an external electrical bias (forward or reverse) is applied, it mainly influences the field distribution within the less photo excited p-i-n photodiode.

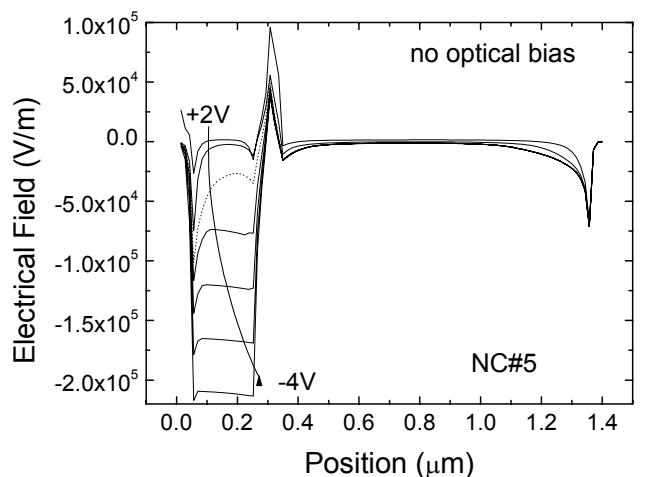

a)

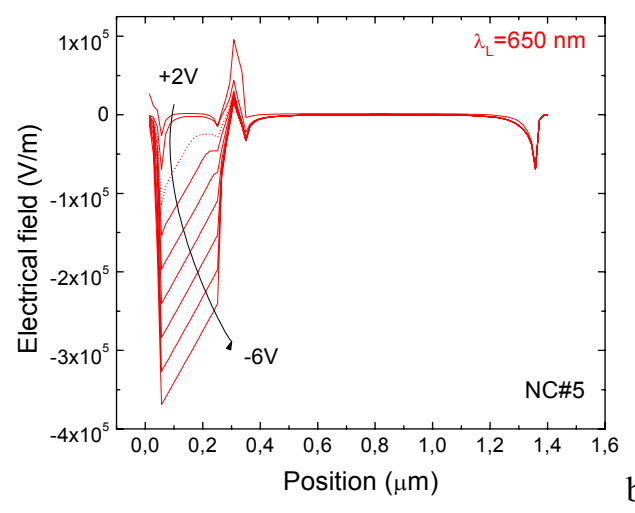

b)

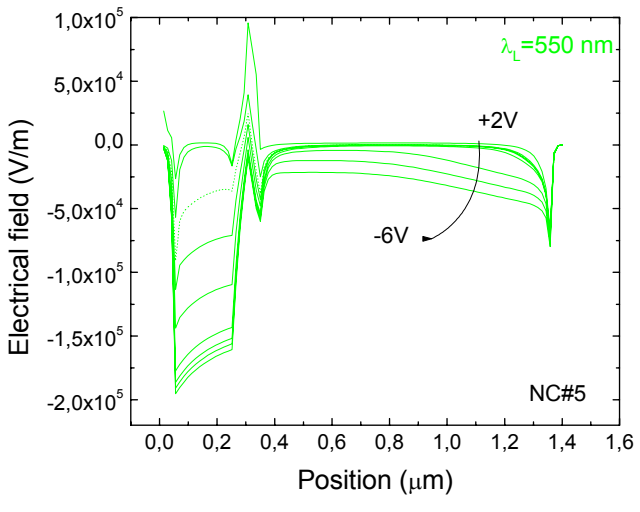

c)

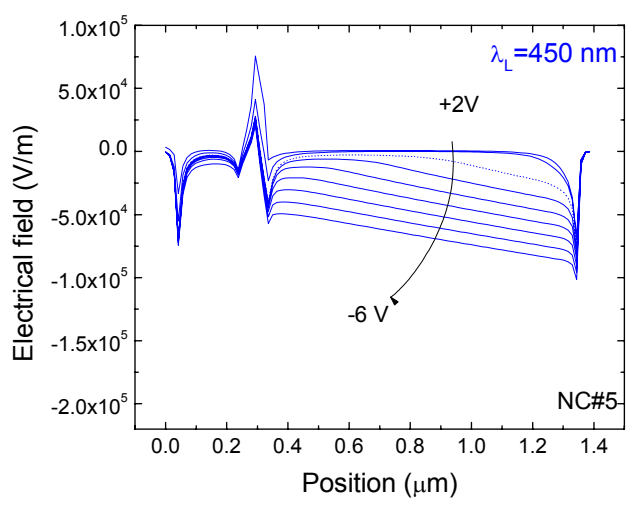

d)

Figure 4 - Electric field profile within the $\mathrm{p}-\mathrm{i}-\mathrm{n} / \mathrm{p}$-i-n tandem structure for different external electrical bias and different wavelengths of impinging light: a) thermodynamic equilibrium .b) $650 \mathrm{~nm}$; c) $550 \mathrm{~nm}$; d) $450 \mathrm{~nm}$. 
When compared with the electric field profile under thermo-dynamical equilibrium conditions, the field under illumination is lowered in the most absorbing cell, while the less absorbing one reacts by assuming a reverse bias configuration. Consequently, opposite behavior is observed under red and blue background light while under green light condition the redistribution of the field profile is balanced between the two sub-cells.

\section{WDM DEVICE OPERATION}

\section{A Wavelength voltage controlled sensitivity}

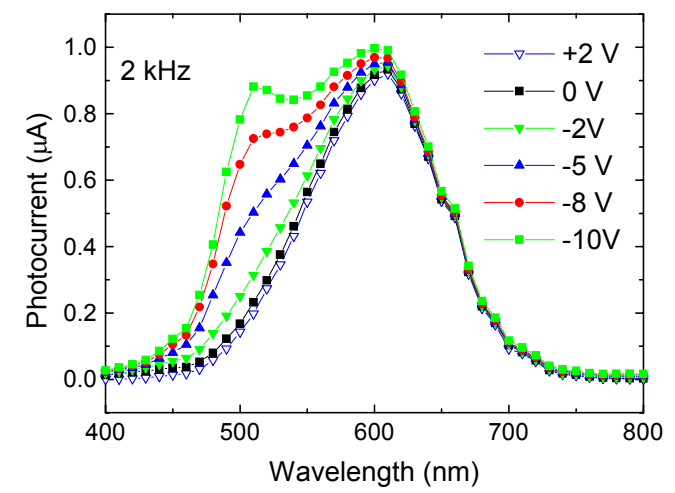

a)

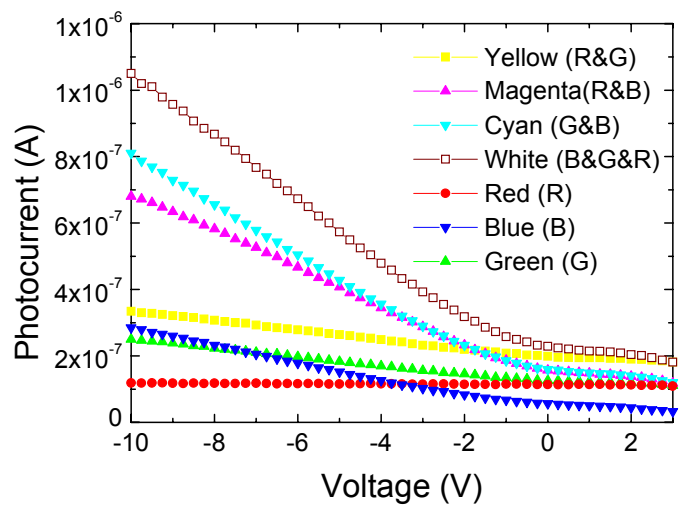

b)

Figure 5 -a) Spectral photocurrent under different applied voltages. b) ac IV characteristics under red (R); green $(\mathrm{G})$ and blue (B), yellow(R\&G); magenta $(R \& B)$; cyan $(G \& B)$ and white $(R \& G \& B)$.

The devices were characterized, at $1 \mathrm{kHz}$, through spectral response $(400-800 \mathrm{~nm})$ and photocurrent-voltage ($10 \mathrm{~V}<\mathrm{V}<+3 \mathrm{~V})$ measurements. In Figure $5 \mathrm{a}$ it is displayed the measured spectral photocurrent and in Figure $5 \mathrm{~b}$ the ac current-voltage characteristics, under illumination, are shown. In this last measurement three modulated monochromatic lights: $\mathrm{R}\left(\lambda_{\mathrm{R}}=650 \mathrm{~nm}\right) ; \mathrm{G}\left(\lambda_{\mathrm{G}}=550 \mathrm{~nm}\right)$ and $\mathrm{B}\left(\lambda_{\mathrm{B}}=450 \mathrm{~nm}\right)$, and their polychromatic combinations; $\mathrm{R} \& \mathrm{G}$ (Yellow); R\&B (Magenta); G\&B (Cyan) and R\&G\&B (White) illuminated separately the device and the photocurrent was measured as a function of the applied voltage.

Results show that as the applied voltage changes from forward to reverse the blue/green spectral collection is enlarged while the red one remains constant (Figure 5a). The photocurrent (Figure 5b) under red modulated light is independent on the applied voltage while under blue, green or combined irradiations, it increases under reverse bias. If the blue spectral component is present (B\&R, $B \& G)$, a sharp increase with the reverse bias is observed. Under positive bias the blue signal becomes negligible and the $R \& B$, the G\&B and the R\&G\&B multiplexed signals overlap, respectively with the $R$, the $G$ and the $R \& G$ signals. This behavior illustrates, under forward bias, the low sensitivity to the blue component of the multiplexed signal. It is interesting to notice that under reverse bias the green signal has a blue-like behavior, while under forward bias its behavior is red-like confirming the green photons absorption across both front and back diodes (Figure 1, Figure 4).

\section{B. $\quad$ Multiplex/ demultiplex modes}

A multiplexer is a device that combines two or more signals onto a single output without losing their specificity. In a simple WDM system, each input channel must emit light at a different wavelength, with all the light channels multiplexed together onto a single optical fiber. After being transmitted through an optical fiber, the combined optical signals must be demultiplexed at the receiving end by distributing the total optical power to each output port and then requiring that each receiver selectively recover only one wavelength by using a tunable optical filter. Each input channel is modulated at a given speed, and the total aggregate capacity being transmitted along the fiber is the sum total of the bit rates of the individual channels.

Multiple monochromatic or a single polychromatic beams are directed to the device where they are absorbed, accordingly to each wavelength (Figure 1), giving rise to a time and wavelength dependent electrical field modulation across it (Figure 4). By reading out, under different applied bias, the total photocurrent generated by all the incoming optical carriers the information (wavelength and Tranmission rate) is multiplexed or demultiplexed and can be transmitted or recovered again. In the multiplexing mode the device faces the modulated light incoming together from different monochromatic channels. The combined effect is converted to an electrical signal via the device. In the demultiplexing mode a single modulated polychromatic light beam (mixture of different wavelength) each one with a different transmission rate (frequency) impinges the device and the spectral sensitivity, which is voltage controlled, allows the recognition of the different color channels. In both modes a programmed logic circuit drive LEDs can be used to send out light into appropriated optical fibers for transmission to a destination where they can be split again using the demultiplexing mode. 


\section{WAVELENGTH DIVISION MULTIPLEXING/DEMULTIPLEXING TECHNIQUES}

\section{A. Bias sensitive WDM multiplexing}
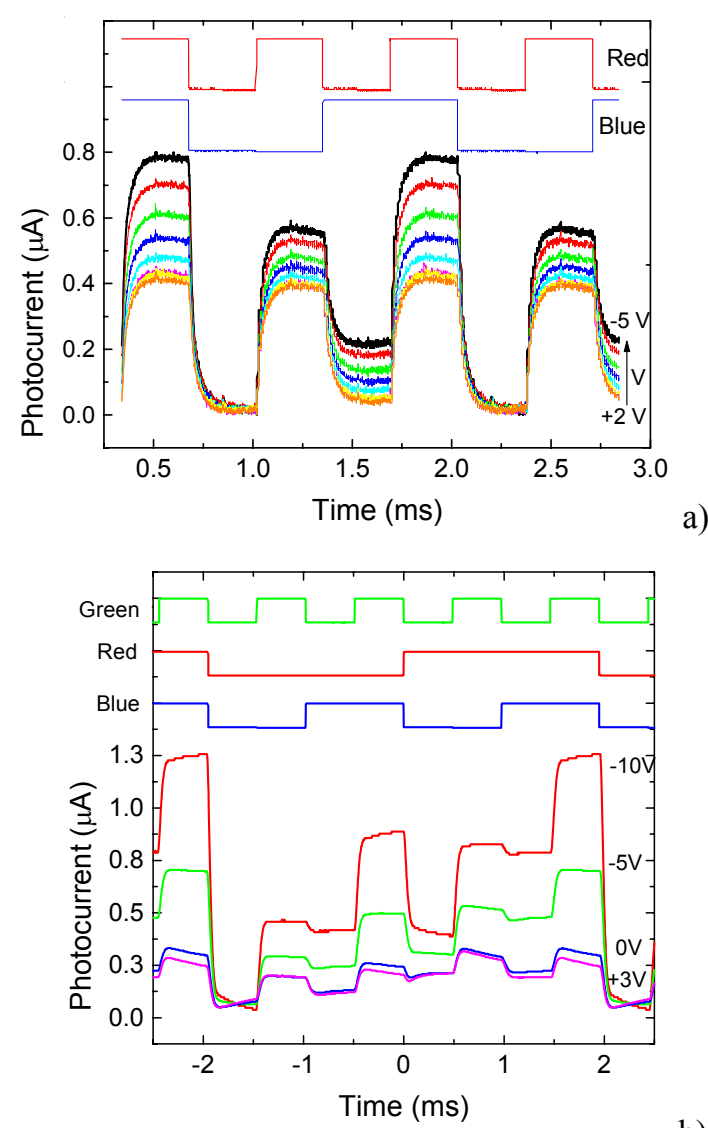

b)

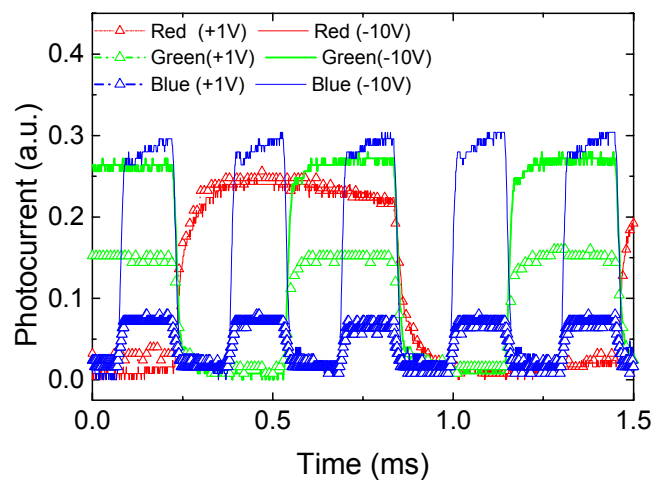

c)

Figure 6 - Transient multiplexed signals at different applied voltages and input wavelengths: a) $R \& B\left(\lambda_{R, B}=650 \mathrm{~nm}, 450 \mathrm{~nm}\right)$. The highest frequency of the input signal is $1.5 \mathrm{kHz}$. b) $R \& G \& B\left(\lambda_{R, G, B}=650 \mathrm{~nm}, 550\right.$ $\mathrm{nm}, 450 \mathrm{~nm}$ ). The highest frequency of the input signal is $1 \mathrm{kHz} c$ ) dependence of the color channel with the applied voltage

The effect of the applied voltage on the output transient multiplexed signal is analyzed. To readout the combined spectra, the generated transient photocurrent due to the simultaneous effect of two $\left(\lambda_{R}=650 \mathrm{~nm}, \lambda_{B}=450 \mathrm{~nm}\right)$ and three $\left(\lambda_{\mathrm{R}}=650 \mathrm{~nm}, \lambda_{\mathrm{G}}=550 \mathrm{~nm}, \lambda_{\mathrm{B}}=450 \mathrm{~nm}\right)$ pulsed monochromatic channels was measured, under different applied voltages. The results are displayed, respectively, in Figure 6a and in Figure 6b. The input wavelength channels are superimposed in the top of the figures to guide the eyes. The reference level was assumed to be the signal when all input channels were OFF (dark level).

In Figure 6a the red frequency was $1.5 \mathrm{KHz}$ and the blue one half of this value while in Figure $6 \mathrm{~b}$ the ratios between the three frequencies were always one half. In Figure $6 \mathrm{c} \mathrm{de}$ dependence of each pulsed single channel with the applied voltage is also displayed. As expected from Figure 2 the red signal remains constant while the blue and the green decrease as the voltage changes from negative to positive. The lower decrease in the green channel when compared with the blue one can be ascribed to the decrease of the photocurrent in the back diode under positive bias (green red-like behavior).

Data show that the multiplexed signal depends on the applied voltage and on the wavelength and transmission rate of the each input channel. Under reverse bias, there are always four (Figure 6a) or eight (Figure 6b) separate levels depending on the number of input channels. The highest level appears when all the channels are $\mathrm{ON}$ and the lowest if they are OFF. Furthermore, the levels ascribed to the mixture of two input channels (R\&B, R\&G, G\&B) are higher than the ones due to the presence of only one (R, G, B). The step among them depends on the applied voltage and channel wavelength. As expected from Figure 5, as the reverse bias increases the signal exhibits a sharp increase if the blue component is present. Under forward bias the blue signal goes down to zero and the red and/or green ones remain constants.

\section{B. Bias sensitive WDM demultiplexing}

Different wavelengths which are jointly transmitted must be separated to regain all the information. These separators are called demultiplexers.

A chromatic time dependent wavelength combination of red and blue (Figure 7a) or red, green and blue (Figure 7b) with different transmission rates, were shining on the device. The generated photocurrent was measured under negative and positive bias to readout the combined spectra. The digital wavelength division demultiplex signals are displayed on the top of both figures.

If only two R and B channels are involved (four levels; Figure 7a), under forward bias, the blue component of the combined spectra falls into the dark level, tuning the red input channel. Thus, by switching between reverse and forward bias the red and the blue channels were recovered and the transmitted information regained. If three $R, G$ and $B$ input channels with different transmission rates are being used (eight levels; Figure 7b), under reverse bias, the levels 
can be grouped into four main thresholds, ascribed respectively to the simultaneous overlap of three (R\&G\&B), two (R\&B, R\&G, B\&G), one ( $R, G, B)$ and none (dark) input channels. Since under forward bias, the blue component of the multiplexed signal approaches the dark level (Figure 5) the R, the $\mathrm{G}$ and the $\mathrm{R} \& \mathrm{G}$ components are tuned. By comparing the multiplexed signals under forward and reverse bias and using a simple algorithm that takes into account the different sub-level behaviors under reverse and forward bias (Figure 6) it is possible to split the red from the green component and to decode their RGB transmitted information.

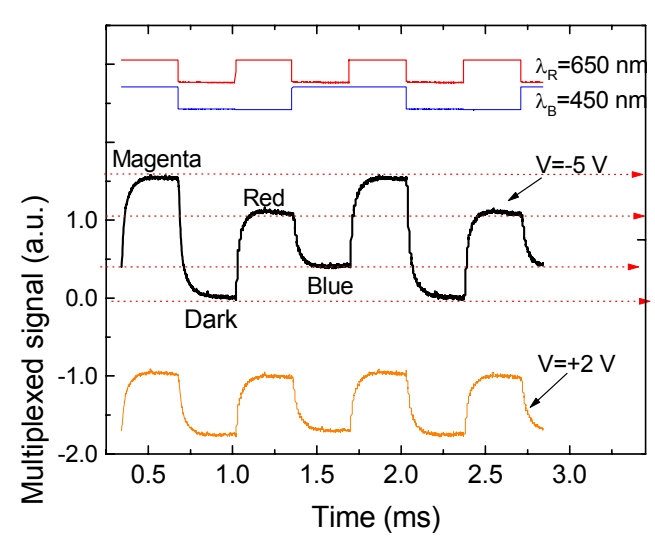

a)

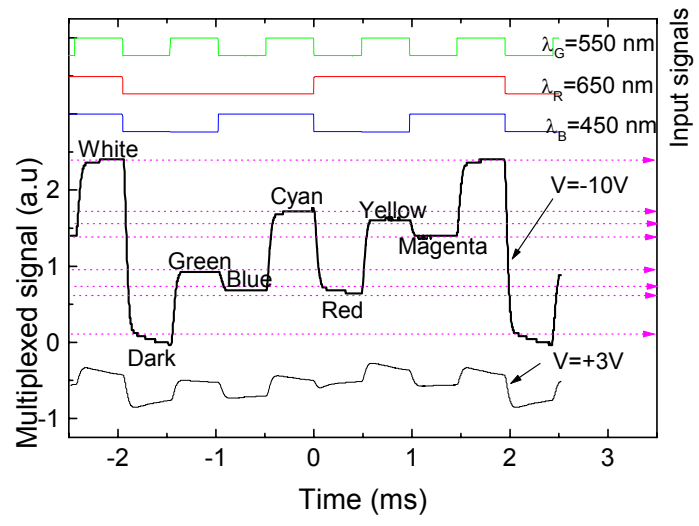

Figure 7 - Transient multiplexed signals under negative and positive bias. a) Polychromatic red and blue time dependent mixture. b) Polychromatic red, green and blue time dependent mixture. The digital wavelength demultiplexed signal is displayed on the top of both figures.

\section{FUTURE TRENDS}

Application fields of polymer optical fibers (POF) technology will increase in future mainly driven by the computing power pervasiveness allied with ubiquitous multimedia home appliances. As a consequence more data transmission capacity will be demanded to the local network infrastructure and at a sustainable cost. The conception of new devices for signal (de)multiplexing in the visible spectrum is an increased demand in this field. To increase the transmission rate $\mathrm{p}-\mathrm{i}-\mathrm{n} / \mathrm{p}-\mathrm{i}-\mathrm{n}$ new WDM devices produced by PECVD, in different architectures, should be tested for a proper fine tuning of the RGB collection efficiency. To test the efficiency of the internal p-n junction, an additional transparent ITO internal contact should be deposited between the $n-p$ internal junctions.

Digital home appliance interfaces, home and car network and traffic control applications are foreseen due to the low cost associated to the a-SiC:H and POF technologies.

\section{VII.CONCLUSION}

Experimental and simulated results on the applicability of a multilayered double $\mathrm{p}-\mathrm{i}-\mathrm{n}$ a-SiC:H/a-Si:H heterostructures, as WDM devices in the visible spectrum, were presented.

Three modulated input channels with different transmission speeds and wavelengths were multiplexed together, without loosing their specificity. The combined optical signal was analyzed by reading out the photocurrent generated across the device. Results show that by switching between positive and negative voltages the input channels could be recovered. A physical model supported by a numerical simulation gives insight into the device operation.

\section{ACKNOWLEDGEMENT}

Authors express thanks to N. Carvalho, and G. Lavareda for sample preparation. This work has been financially supported the research project PTDC/ENR/67616/2006.

\section{REFERENCES}

[1] Manabu Kagami. "Optical Technologies for Car Applications Innovation of the optical waveguide device fabrication". Optical communications - perspectives on next generation technologies, October 23-25, 2007 in Tokyo, Japan.

[2] M. Haupt, C. Reinboth and U. H. P. Fischer. "Realization of an Economical Polymer Optical Fiber Demultiplexer", Photonics and Microsystems, 2006 International Students and Young Scientists Workshop, Wroclaw, 2006.

[3] S. Randel, A.M.J. Koonen, S.C.J. Lee, F. Breyer, M. Garcia Larrode, J. Yang, A. Ng'Oma, G.J Rijckenberg, H.P.A. Boom. "Advanced modulation techniques for polymer optical fiber transmission". proc. ECOC 07 (Th 4.1.4). Berlin, Germany (2007) 1-4.

[4] M. Haupt, C. Reinboth and U. H. P. Fischer. "Realization of an Economical Polymer Optical Fiber Demultiplexer", Photonics and Microsystems, 2006 International Students and Young Scientists Workshop, Wroclaw, 2006.

[5] M. Vieira, A. Fantoni, M. Fernandes, P. Louro, G. Lavareda and C.N Carvalho, Thin Solid Films, 515, Issue 19, 2007, 7566-7570.

[6] C. Nunes de Carvalho, G. Lavareda, A. Amaral, O. Conde and A. R. Ramos, J. Non-Cryst. Solids 352, Issues 23-25, (2006), p. 2315

[7] A. Fantoni, M. Vieira, R. Martins, Mathematics and Computers in Simulation, Vol. 49. (1999) 381-401

[8] P. Louro, M. Vieira, Yu. Vygranenko, A. Fantoni, M. Fernandes, G. Lavareda, N. Carvalho Mat. Res. Soc. Symp. Proc., 989 (2007) A12.04.

[9] E. A. Schiff, R. A. Street, R. L. Weisfield, J. Non-Cryst. Solids 198200 (1996) 1155. 